\title{
Related graphs of the conjugacy classes of a 3-generator 5-group
}

\author{
Alia Husna Mohd Noor*, Nor Haniza Sarmin, Hamisan Rahmat \\ ${ }^{a, b}$ Department of Mathematical Sciences, Faculty of Science, Universiti Teknologi Malaysia, 81310 UTM Johor Bahru, Johor, Malaysia \\ *Corresponding author: mylife_syafia@yahoo.com
}

\section{Article history}

Received 3 May 2018

Revised 25 August 2018

Accepted 11 September 2018

Published Online 25 October 2018

\begin{abstract}
The study on conjugacy class has started since 1968. A conjugacy class is defined as an equivalence class under the equivalence relation of being conjugate. In this research, let $G$ be a 3-generator 5group and the scope of the graphs is a simple undirected graph. This paper focuses on the determination of the conjugacy classes of $G$ where the set omega is the subset of all commuting elements in the group. The elements of the group with order 5 are identified from the group presentation. The pair of elements are formed in the form of $(a, b)$ which is of size two where $a$ and $b$ commute. In addition, the results on conjugacy classes of $G$ are applied into graph theory. The determination of the set omega is important in the computation of conjugacy classes in order to find the generalized conjugacy class graph and orbit graph. The group action that is considered to compute the conjugacy classes is conjugation action. Based on the computation, the generalized conjugacy class graph and orbit graph turned out to be a complete graph.
\end{abstract}

Keywords: Conjugacy class, Generalized conjugacy class graph, Orbit graph

\section{INTRODUCTION}

A conjugacy class can be defined as an equivalence class under the equivalence relation of being conjugate. Besides, a conjugacy class of a group is a set of elements under the action of the group on itself by conjugation.

The study on conjugacy class has started many years ago by Erdos and Turan (Erdos, P. and Turan, P., 1968) who considered on the conjugacy class of symmetric groups, $S_{n}$. In 2013, Al-Hasanat, et al. (Al-Hasanat, B. N. et al., 2013) found the conjugacy class of dihedral groups of order $2^{m+1}$.

Many researchers are interested in relating the study on group theory and graph theory since the research on conjugacy class has been expanding for several years. For example, Ilangovan (Ilangovan, S., 2013) applied the results from the computation of the conjugacy classes of the groups of nilpotency class 2 into graph theory.

In this paper, the conjugacy class of 3 -generator $p$-group is computed where $p=5$. The group presentation is taken from Burnside (Burnside, W., 1897). Burnside studied on theory of groups of finite order. In his research, there are 15 types of group presentation of groups of order $p^{n}$ where $n=4$. In this research, we only considered the groups with three generators. The relations among the generators are differents between each group. The group presentations are stated as follows:

$$
\begin{gathered}
H_{1}=\langle x, y, z| x^{p}=y^{p}=z^{p^{2}}=1,[x, z]=[y, z]=1,[x, y]=z^{p}>, \\
H_{2}=\left\langle x, y, z \mid x^{p^{2}}=y^{p}=z^{p}=1,[x, z]=[y, z]=1, x^{y}=x^{3}\right\rangle, \\
H_{3}=\left\langle x, y, z \mid x^{p^{2}}=y^{p}=z^{p}=1,[x, z]=[y, z]=1,[x, y]=z\right\rangle .
\end{gathered}
$$

However, in this paper, we only consider one group presentation which is the first group since the computation of conjugacy class is completed only for $H_{1}$ and we represent the group as $G$.

$$
G=<x, y, z \mid x^{p}=y^{p}=z^{p^{2}}=1,[x, z]=[y, z]=1,[x, y]=z^{p}>,
$$

A conjugacy class graph consists of vertices and edges where the vertices are represented by the non-central conjugacy classes of a group $G$. Meanwhile, two vertices are connected and form edges if the greatest common divisor of the order of vertices are not equal to one.

There are some researches done on conjugacy class graph. In 2016, Ibrahim, et al.( Ibrahim, A. A. et al., 2016) worked on the conjugacy classes of some finite metabelian groups and their related graph. Alimon, et al.(Alimon, N. I. et al., 2018) also studied on conjugacy class graph where they found the energy of four type of graphs including the conjugacy class graph. The group considered in their paper is some metacyclic 2-groups.

In this paper, we want to determine the generalized conjugacy class graph and orbit graph. Hence, the condition which are needed to be satisfied in order to find both of the graph for the group $G$ is mentioned in the following:

Let $G$ be a finite group. Let $\Omega$ be the set of all ordered pairs $(a, b)$ in $G \times G$ such that $\operatorname{lcm}(a, b)=p, a b=b a$ and $a \neq b$. The group action of $G$ on $\Omega$ is by conjugation action.

This paper is divided into three parts. The first part is the introduction about conjugacy class and group presentation. Some definitions on graph theory are presented in the second part which 
include the generalized conjugacy class graph and orbit graph. The results on the conjugacy class and graphs are explained in the third part.

\section{PRELIMINARIES}

In this section, some basic knowledge on graphs related to conjugacy class are presented.

\section{Definition 1 (Fraleigh, J. B. 2000)}

Let $G$ be a finite group and $g$ be the element in $G$. The conjugacy class of $g$ is written as $c l(g)=\left\{x^{-1} g x \mid x \in G\right\}$. The number of conjugacy class is denoted by $K(G)$.

\section{Definition 2 (Marcus, D. A. 2008)}

A graph consist of points, which are called vertices and connections, which are called edges and which are indicated by line segments of curves joining certain pairs of vertices.

\section{Definition 3 (Marcus, D. A. 2008)}

If all of the vertices in a graph are adjacent to each other, then the graph is called a complete graph. The symbol $K_{n}$ is used to denote a complete graph with $n$ vertices.

\section{Definition 4 (Machale, D. 1974)}

The graph $\Gamma$ is an empty graph, if there is no adjacent (edges) between it vertices, which is denoted as $K_{e}$.

\section{Definition 5 (El-Sanfaz, M. A. and Sarmin, N. H. 2015)}

Let $G$ be a finite group. Let $\Omega$ be the set of all ordered pairs $(a, b)$ in $G \times G$ such that $\operatorname{lcm}(a, b)=p, a b=b a$, and $a \neq b$. If $G$ acts on $\Omega$ by conjugation, the number of the vertices of generalized conjugacy class graph are

$$
\left|V\left(\Gamma_{G}^{\Omega}\right)\right|=K(\Omega)-|A|
$$

where $K(\Omega)$ is the number of conjugacy classes under group action on $\Omega$ and $A=\{w \in \Omega, g w=w g, g \in G\}$. Two vertices $w_{1}$ and $w_{2}$ are adjacent if their cardinalities are not coprime, i.e $\operatorname{gcd}\left(\left|w_{1}\right|,\left|w_{2}\right|\right) \neq 1$.

Definition 6 (Omer, S. M. S., Erfanian, A. and Sarmin, N. H. 2015)

Let $G$ be a finite group and $\Omega$ is a set of $G$. Let $A$ be the set of commuting elements in $G$ i.e $A=\{w \in \Omega, g w=w g, g \in G\}$. The orbit graph $\Gamma_{G}^{\Omega}$, consists of two sets, namely vertices and edges denoted by $V\left(\Gamma_{G}^{\Omega}\right)$ and $\mathrm{E}\left(\Gamma_{G}^{\Omega}\right)$, respectively. The vertices of $\Gamma_{G}^{\Omega}$ are non-central elements in $\Omega$ but not in $A$, that is $\left|V\left(\Gamma_{G}^{\Omega}\right)\right|=\Omega-|A|$. Two vertices $v_{1}$ and $v_{2}$ are adjacent if the following conditions are satisfied:

1. If there exists $g \in G$ such that $g v_{1}=v_{2}$.

2. If the vertices $\Gamma_{G}^{\Omega}$ are conjugate, that is $v_{1}=g^{v_{2}}$.

\section{MAIN RESULTS}

In this section, the main results on the generalized conjugacy class graph and orbit graph of the 3-generator 5-group are presented.

\section{Proposition 1}

Let $G=<x, y, z \mid x^{p}=y^{p}=z^{p^{2}}=1,[x, z]=[y, z]=1,[x, y]=z^{p}>$, be a finite group. Let $\Omega$ be the set of all ordered pairs $(a, b)$ in $G \times G$ such that $\operatorname{lcm}(a, b)=5, a b=b a$ and $a \neq b$. If $G$ acts on $\Omega$ by conjugation, then the number of conjugacy classes of $G$ is 716 .

Proof:

$G$ consists of an identity and 124 elements of order 5. From the 125 elements, 1750 pairs of element are formed based on the given case which is known as $\Omega$. The pair of elements are starting with

$$
(1, x),\left(1, x^{2}\right), \ldots,\left(x^{4} y^{4} z^{5}, x^{4} y^{4} z^{10}\right) .
$$

By using Definition 1, the conjugacy classes for each pair of element are computed. Some conjugacy classes obtained are listed as follows;

$$
\begin{aligned}
& \text { 1.cl }(1, x)=\left\{(1, x),\left(1, x z^{5}\right),\left(1, x z^{10}\right),\left(1, x z^{15}\right),\left(1, x z^{20}\right)\right. \\
& \text { 2.cl }\left(1, x^{2}\right)=\left\{\left(1, x^{2}\right),\left(1, x^{2} z^{5}\right),\left(1, x^{2} z^{10}\right),\left(1, x^{2} z^{15}\right),\left(1, x^{2} z^{20}\right) .\right.
\end{aligned}
$$

$$
\begin{aligned}
& \text { 9.cl }\left(1, z^{5}\right)=\left\{\left(1, z^{5}\right)\right\} \\
& 10 . c l\left(1, z^{10}\right)=\left\{\left(1, z^{10}\right)\right\}
\end{aligned}
$$

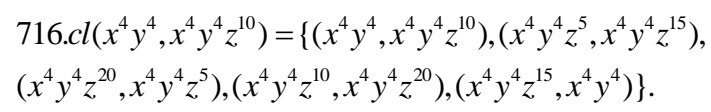

The conjugacy classes that contain only one pair of element is considered as central conjugacy class. In this computation, there are 20 central conjugacy classes and 696 pairs of element with 5 elements in each conjugacy class. Therefore, the number of conjugacy classes of $G$ is 716 .

\section{Proposition 2}

Let $G=<x, y, z \mid x^{p}=y^{p}=z^{p^{2}}=1,[x, z]=[y, z]=1,[x, y]=z^{p}>$, be a finite group. Let $\Omega$ be the set of all ordered pairs $(a, b)$ in $G \times G$ such that $\operatorname{lcm}(a, b)=5, a b=b a$ and $a \neq b$. If $G$ acts on $\Omega$ by conjugation, then the generalized conjugacy class graph of $G, \Gamma_{G}^{\Omega}=K_{696}$. Proof:

Applying Definition 5 into this proposition, the number of vertices of generalized conjugacy class graph is 696 since the number of the set of commuting elements in $G$ is 20 .

Consider the conjugacy classes below which are among the 696 conjugacy classes obtained from the computation;

$$
\begin{aligned}
& c l\left(x, x^{2}\right)=\left\{\left(x, x^{2}\right),\left(x z^{5}, x^{2} z^{10}\right),\left(x z^{10}, x^{2} z^{20}\right),\left(x z^{15}, x^{2} z^{5}\right),\left(x z^{20}, x^{2} z^{15}\right)\right\} \\
& c l\left(x, x^{3}\right)=\left\{\left(x, x^{2}\right),\left(x z^{5}, x^{3} z^{15}\right),\left(x z^{10}, x^{3} z^{5}\right),\left(x z^{15}, x^{3} z^{20}\right),\left(x z^{20}, x^{3} z^{10}\right)\right\} \\
& c l\left(x, x^{4}\right)=\left\{\left(x, x^{4}\right),\left(x z^{5}, x^{4} z^{20}\right),\left(x z^{10}, x^{4} z^{15}\right),\left(x z^{15}, x^{4} z^{10}\right),\left(x z^{20}, x^{4} z^{5}\right)\right\}
\end{aligned}
$$

It can be seen that, each conjugacy class consists of five pair of elements with the same order. The cardinalities between each vertices are not coprime i.e

$$
\begin{aligned}
& \operatorname{gcd}\left\{\left(x, x^{2}\right),\left(x, x^{3}\right)\right\}=\operatorname{gcd}\{(5,5)\}=5 \\
& \operatorname{gcd}\left\{\left(x, x^{2}\right),\left(x, x^{4}\right)\right\}=\operatorname{gcd}\{(5,5)\}=5
\end{aligned}
$$

Thus, all vertices are connected and the generalized conjugacy class graph of $G$ is a complete graph with 696 vertices, $K_{696}$. 


\section{Proposition 3}

Let

$G=<x, y, z \mid x^{p}=y^{p}=z^{p^{2}}=1,[x, z]=[y, z]=1,[x, y]=z^{p}>$, be a finite group. Let $\Omega$ be the set of all ordered pairs $(a, b)$ in $G \times G$ such that $\operatorname{lcm}(a, b)=5, a b=b a$ and $a \neq b$. If $G$ acts on $\Omega$ by conjugation, then the orbit graph of $G, \Gamma_{G}^{\Omega}=\bigcup_{i=1}^{696} K_{5}$.

Proof:

The number of vertices of the orbit graph is 696 since the number of conjugacy classes is 716 and the number of $A$ is 20 . Based on the computation in Proposition 1, each conjugacy class has five pairs of element. Hence, condition 2 in Definition 6 is satisfied where the vertices in the orbit graph are conjugate to each other. Thus, the orbit graph, $\Gamma_{G}^{\Omega}=\bigcup_{i=1}^{696} K_{5}$.

\section{CONCLUSION}

In conclusion, the generalized conjugacy class graph of $G$ is a complete graph with 696 vertices. Meanwhile, the orbit graph of $G$ turned out to be the union of complete graphs of degree 5 .

\section{ACKNOWLEDGEMENT}

The first author would like to express her appreciation to Universiti Teknologi Malaysia for the Research University Grant (GUP) for Vote No. 13J82.

\section{REFERENCES}

Al-Hasanat, B. N., Almatroud, O. A. and Ababneh, M. S. Dihedral groups of order $2^{m+1}$. International Journal of Applied Mathematics. 26(1), $1-7,2013$.

Alimon, N. I., Sarmin, N. H., Ahmad Fadzil, A. F. The energy of four graphs of some metacyclic 2-groups. Malaysian Journal of Fundamental and Applied Sciences. 14(1), 59 - 66, 2018.

Burnside, W. Theory of Groups of Finite Order. $7^{\text {th }}$ ed. Cambridge: University Press. 1897.

Erdos, P. and Turan, P. On some problems of a statistical group theory. IV. Acta. Math. Acad. Sci. Hung. 19(3-4), 413 - 435, 1968.

El-Sanfaz, M. A. and Sarmin, N. H. On the probability that an element of metacyclic 2-group of positive type fixes a set and its generalized conjugacy class graph. Global Journal of Pure and Applied Mathematics. 11(2), 899-908, 2015.

Fraleigh, J. B. A First Course in Abstract Algebra. $7^{\text {th }}$ ed. USA: Addison Wesly Longman, Inc. 2000.

Ilangovan, S. Conjugacy classes and graphs of two-groups of nilpotency class 2. Ph.D Thesis. Universiti Teknologi Malaysia. 2013.

Ibrahim, A. A., Sarmin, N. H., Mohd Noor, A. H. and Omer, S. M. S. The conjugacy classes of some finite metabelian groups and their related graphs. Jurnal Teknologi. 79(1), 69-73, 2017.

Marcus, D. A. Graph theory: A problem oriented approach. The Mathematical Association of America. Washington. 2008.

MacHale, D. How commutative can a non-commutative group be? The Mathematical Gazette. 58(405), 199 - 202, 1974.

Omer, S. M. S., Erfanian, A. and Sarmin, N. H. The orbit graph of finite non-abelian groups. International Journal of Pure and Applied Mathematics. 102(4), 745 - 755, 2015. 